\title{
EDITORIAL
}

\section{Tissue Transplantation for Parkinson's Disease}

\author{
Donald B. Calne and Patrick L. McGeer
}

In 1987, Madrazo et al $^{1}$ reported spectacular improvement in parkinsonian patients subjected to transplantation of adrenal medullary tissue into the caudate nucleus. As of August 1988, over 200 parkinsonian patients have undergone similar procedures. The human experience far exceeds that on non-human primates. From this background there has emerged a large number of verbal anecdotal reports, a considerable number of newspaper, television and radio statements, a small number of communications presented to scientific meetings, and hardly any peer reviewed reports in the medial literature. ${ }^{2}$ In general, the confusion generated in this type of situation can be tolerated. However, in the current context, there are unusual problems. No mechanisms exist to coordinate or control experimental surgery. Meanwhile at least forty centers are involved in transplant surgery on the brain, some operating in private practice. Furthermore, experimental work is extending to the use of fetal tissue. This raises a set of ethical questions not previously dealt with in any medical context. Under the circumstances, it is desirable to pause and review the situation. What conclusions, if any, can be drawn from the conglomeration of accounts of the results of the world experience of transplanting tissue into the brain of parkinsonian patients? What directions are appropriate for the future? Canadian interest in this subject was evident in two symposia held in June in Quebec City. One was initiated by the Movement Disorder Group of Canada, the other by the Canadian Association of Anatomists. The issues arising from these symposia can best be addressed by formulating specific questions about the human brain transplantation experience to date.

1. Have the results reported from Mexico in 1987 been replicated? So far, no center with specialized experience in the evaluation of Parkinson's disease has been able to confirm the highly successful claims that caught the attention of the world when they were published in the New England Journal of Medicine last year.

2. Has some efficacy from transplantation procedures nevertheless been unequivocally established? The answer to this question is uncertain. Many centers with expertise in Parkinson's disease consider that they have observed a reduction in neurological deficits following surgery. The problem is that the experiments have not been designed with comparable rigor to that demanded in pharmacotherapy trials. Parkinson's disease is known to display marked fluctuations in severity and puzzling changes according to circumstances (kinesia paradoxica). Neurologists are all too familiar with this difficulty. Reports of improved movement in some patients on the side ipsilateral to the brain transplant has been viewed by some in the context of a placebo response. No comparable effect has ever been reported in animal experiments. Some placebo response must be anticipated in circumstances where a major neurosurgical procedure is associated with high media profile, and where repeated specialized patient-physician interaction must take place. These factors create uncertainty about the positive results reported.

3. Have transplantation operations been shown to be safe? While the complications of surgery have varied from center to center, the general experience has included a significant incidence of major problems, including aspiration pneumonia, frontal infarction, the neuroleptic malignant syndrome, and death. The parkinsonian patient population has not been made properly aware of these serious hazards since media attention has focused on the dazzling claims of success.

4. Is the risk-benefit ratio acceptable? Opinions on this question will vary widely because of the paucity of reliable data concerning both risks and benefits. If the immediate risk to life of any disease is high, morbidity and mortality will be accepted as inevitable consequences of an experimental procedure. For example, in the surgical field, heart and lung transplants were initially undertaken on patients with very limited life expectancy. Positive results in some of those cases were regarded as miraculous. But this is not the situation for the average parkinsonian patient. There is a reasonable prognosis on conventional medical treatment. Therefore the risks must be less and the benefits greater than with other, more life threatening situations.

Experimental surgery has never been subjected to the same discipline required in the field of pharmacotherapy. New drug regimens go through phases of trial in which risks and benefits are systematically monitored. Investigators require prior clearance from appropriate authorities before undertaking a new drug trial and the results are carefully evaluated by government agencies. Permission must even be sought from national regulatory authorities before an experimental drug can be provided on an individual emergency basis. No such surgical restraints exist. The uncontrolled proliferation of centers attempting brain transplantation for Parkinson's disease is totally in conflict to soci-

Reprint requests to: D.B. Calne, Department of Medicine, Division of Neurology, University Hospital, 2211 Wesbrook Mall, Vancouver, B.C., Canada V6T IW5 
ety's demand for independent control in the development of new medications. No drug would be marketed on the basis of the currently demonstrated efficacy and safety of brain transplant procedures. Indeed, government regulatory agencies might even terminate investigational studies.

5. Has there been resolution of the ethical issues deriving from transplantation of fetal tissue? Ethical concerns relating to transplant surgery have only been satisfactorily resolved for living donors or cadavers where informed consent has been obtained from living relatives. In the very limited context of fundamental research, fetal tissue has been treated in the same fashion as tissue from adult cadavers. But fetal tissue is different in that therapeutic abortions are voluntary and pregnancy could be undertaken with a view to procuring tissue. This creates new ethical dilemmas which are by no means trivial.

If the tissues themselves have therapeutic value, they will be sought on an anticipatory basis. The problem could reach distressing proportions if the potential demand far exceeds the foreseeable supply. Since the prevalence rate of Parkinson's disease is of the order of 1 in 200 persons over the age of 60 years and the salvage of appropriate fetal nigral cells at abortion is marginal, this could become a significant problem. Clearly, ethicists, religious leaders and governments must render a consensus on what guidelines should apply. In the meantime, the U.S. Government has banned NIH participation in human fetal transplants and the Honorable Jacob Epp has placed a comparable ban in Canada for government funded research on any tissue from an elective abortion.

6. Have the experimental procedures currently being used in humans been adequately tested at the animal experimental level? Grafts of the type undertaken by Madrazo and colleagues, where clips were used to fasten adrenal tissue to the caudate nucleus, had never been undertaken in animals. There was, therefore, no firm base in fundamental neuroscience against which the prospects of survival of the grafted tissue for any length of time could be judged despite extensive experience with autografts, heterografts and even zenografts into the CNS. It would be fair to say that the clinical procedures used in human transplants took neuroscientists by surprise. If many more human cases are undertaken, it will soon be known if the procedure is safe for animals.

\section{Conclusions}

There are inadequate answers to most of the questions that demand attention in order to formulate a rational policy on tis- sue transplantation for Parkinson's disease. Nevertheless, practical decisions have to be made. One course would be to advocate no action pending additional results from transplants that have already been performed. A voluntary agency for accumulating data is functioning in Chicago under the aegis of the American Association of Neurosurgeons. Another approach would be to recommend total and permanent termination of transplantation procedures on the grounds of evident risk and questionable benefit. We reject these extremes and suggest a middle course. There is reason from basic science research for believing that transplantation offers some potential for the future. To snuff out all work because of an injudicious clinical start might cut off an avenue of justifiable hope for all those parkinsonian patients waiting for better methods of therapy. On the other hand, there is unequivocal evidence that brain transplant surgery is being pushed too hard. Patients must be made aware of the serious risks that accompany current procedures. Studies must be designed in which transplantation is compared with an alternative form of treatment in a setting where assessment of efficacy can be undertaken by "blind" evaluators. There is an immediate need for government agencies to begin working in cooperation with the medical profession, basic scientists and ethical authorities to draft guidelines that can be adapted to experimental surgery in general. The same applies to the use of fetal tissue for therapeutic purposes.

The use of fetal tissue will be a continuing ethical problem. That is why, for the moment at least, the promising area of transplanting cultured cells which can be provided in abundance, deserves to be vigorously explored. The guidelines used for introduction of new pharmacotherapeutic agents might be a starting point for formulating future policy, although no one would wish for an extension of the bureaucratic delays that may be associated with the licensing of new drugs. As far as basic science is concerned, the premature application of results to the human situation speaks volumes with respect to the desirability of expanding research in the field of transplantation.

\section{REFERENCES}

1. Madrazo I, Drucker-Colin R, Diaz V, Martinez-Mata J, Torres C, Becerril JJ. Open microsurgical autograft of adrenal medulla to the right caudate nucleus in two patients with intractable Parkinson's Disease. New Eng J Med 1987; 316 (14): 831-874.

2. Joynt RJ, Gash DM. Neural Transplants: Are we ready? Ann Neurol 1987; 22 (4); 455-456. 